\title{
A Precision Six-load-component Transducer: A Design Incorporating Finite-length Measurement Paths
}

\author{
by Peter $\mathrm{D}$. Washabaugh
}

\begin{abstract}
The design of an instrument is described that measures three resultant force components and three resultant moment components acting on a surface. Within the framework of linear elastostatics of an isotropic homogeneous material the device separates to a given precision the six resultant load components. Sensor paths of finite length are employed. Moreover if fiber-optic differential displacement sensors are used rather than traditional electrical resistance strain gages, the range and sensitivity of the instrument can in principle be improved without sacrificing the device stiffness. The primary reason for these improvements is that a complete solution to the equations of elasticity allows certain displacements to be measured over large distances and be combined to yield all of the resultant load components. These displacement measurements over a long distance accommodates the use of fiber-optic interferometric sensors. The use of optical sensors in contrast with electrical-resistance gages, has the potential to allow the measurement precision and range to scale with the geometry of the device rather than the maximum strain in the instrument. It becomes possible by virtue of these features to produce a better instrument.
\end{abstract}

\section{Introduction}

It is generally desirable to have a load-measuring instrument that is rigid and inherently free of calibration requirements. For a given load, a stiff instrument is less invasive than a more flexible counterpart, in the sense that less energy is required from the measured system. Reducing calibration requirements lowers the number of intrinsic states of a device. This simplification makes the instrument more elemental and thereby increases the measurement confidence.

Peter D. Washabaugh is Assistant Professor of Aerospace Engineering, University of Michigan, College of Engineering, Aerospace Engineering Building, Ann Arbor, MI 48109-2140.

Original manuscript submitted: January 21, 1992. Final manuscript received: February 22, 1993
Typical multicomponent load-measuring instruments have some means to generate a response within the device that is particular to one of the desired load components. Frequently the device has a small area that is made sensitive to a single component of the applied load by mechanical means. This mechanical separation of the components is usually accomplished by weakening the instruments's structure along certain paths. ${ }^{1,2}$ These weakened locations not only provide some separation of the various components, but due to their higher strain make ideal places to mount electrical strain gages. ${ }^{3}$

Unfortunately this methodology has several disadvantages. Increasing the strain in the instrument decreases the overall stiffness and typically precludes its use as a primary or critical load-bearing structure. Further, most transducers frequently retain significant sensitivity to off design loading conditions. A way to correct for this crosstalk is to calibrate the instrument in a combined loading environment and compensate for the undesired properties by external circuitry. The inspiration for this investigation comes from recognizing that typical multicomponent load transducers ${ }^{1,2,4}$ rely on structural-mechanics concepts such as Bernoulli-Euler beam theory or Kirchhoff plate theory ${ }^{5}$ for their design. The present goal is to investigate an instrument's design if the more refined strains of a complete solution to the equations of elasticity are utilized. In particular the intention is to determine means to eliminate cross-talk in sensing load components and improve the overall stiffness, range, and precision of the instrument.

Here a methodology is described for the construction of a multicomponent load transducer that makes several improvements on conventional designs. Within the framework of linear elastostatics of an isotropic homogenous material, and one-dimensional strain or displacement sensors, this transducer alleviates some calibration problems by separating the signals of all the six resultant load components to a desired precision. The use of a complete solution allows the load-component separation to be maintained even for long sensor paths. 


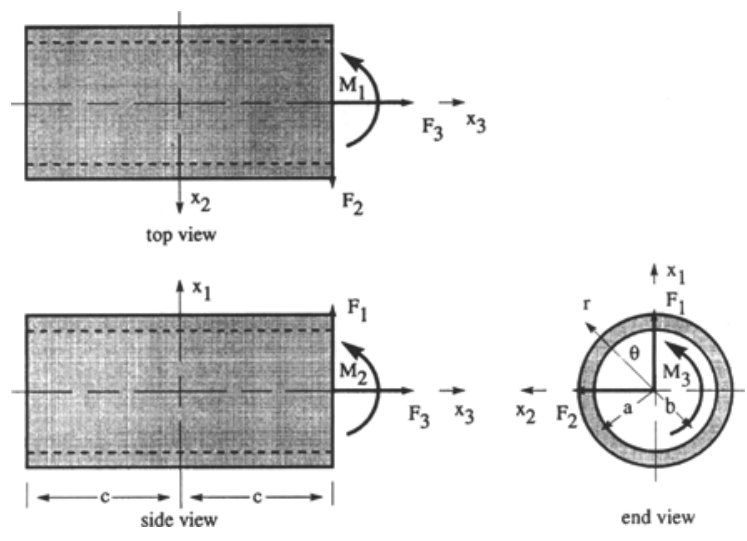

Fig. 1-Schematic of a circular cylinder and the associated Cartesian and cylindrical coordinate systems along with the resultant load components acting on the positive face of the cylinder

The protracted sensor paths accommodates the use of displacement sensors such as fiber-optic Mach-Zehnder interferometers. ${ }^{6}$ Using displacement sensors in lieu of electrical-resistance strain gages allows the measurement range and precision to be scaled with the geometry of the transducer rather than the maximum strain. Explicitly removing the strain from the measurement scaling has the potential to allow a stiffer instrument without sacrificing range or sensitivity. Further, reducing the maximum strain admits the use of the instrument as a primary load-bearing element.

\section{A Complete Solution in Elastostatics: A Cylinder with End Loads}

There are several exact solutions of finite regions with arbitrary loads in three-dimensional elasticity. For example, prismatic structures with either rectangular or elliptical cross-sections and tractions on the end faces have been solved. ${ }^{7}$ A prismatic tube with a circular section was chosen for this investigation for the simple reason that under pure torsion the cross-section does not warp. ${ }^{7}$ A hollow tube is employed to give some flexibility in scaling the geometry.

Consider a prismatic circular tube of outside radius $a$, inside radius $b$, and half length $c$ as shown in Fig. 1. The axis of the cylinder is aligned with the $x_{3}$ direction of a Cartesian coordinate system $\left(x_{1}, x_{2}, x_{3}\right)$. For convenience a typical cylindrical coordinate system $\left(r, \theta, x_{3}\right)$ is also defined.

The cylinder is assumed to be in static equilibrium. The positive face of the cylinder, at $x_{3}=c$, is loaded by arbitrary surface tractions. The lateral surfaces, $r=a$ and $r=b$ are not loaded. The cylinder is further loaded by a constant gravitational acceleration, $g_{3}$, in the $x_{3}$ direction, and strained by a uniform change in temperature $\Delta T$, measured from ambient. The negative end of the cylinder, at $x_{3}=-c$, has the surface tractions necessary to maintain static equilibrium.
Integrating the arbitrary surface tractions on the positive face of the cylinder yields the resultant or statically equivalent loads. The three resultant force components due to the applied surface tractions on the $x_{3}=c$ face are denoted by $F_{i}$ where $i(i=1,2,3)$ indicates the component direction. The three resultant moment components on the same face are similarly denoted by $M_{i}$, assuming the moment is positive in the direction of the Cartesian coordinate system using a right-hand rule.

The cylinder is assumed to be composed of an isotropic, homogeneous, linear elastic material. Let $E$ denote the Young's modulus, $v$ the Poisson ratio, $\rho$ the density, and $\alpha$ the coefficient of thermal expansion of the material. This problem can be made nondimensional as given in eq (1). For a particular set of surface tractions, the components of the Lagrangian strain tensor in the Cartesian coordinate system, throughout the entire cylinder, are given by eq (2). ${ }^{7}$ Equation (3) defines terms that are used to make the strain equations more compact. Note that $\hat{A}, I, J$, and $\hat{G}$ are nondimensional versions of the cross-sectional area, moment of inertia, torsional constant, and the shear modulus, respectively.

$$
\begin{aligned}
& \hat{a}=1 \quad \hat{b}=\frac{b}{a} \quad \hat{c}=\frac{c}{a} \quad \hat{x}_{i}=\frac{x_{i}}{a} \\
& \hat{F}_{i}=\frac{F_{i}}{E a^{2}} \quad \hat{g}_{3}=\frac{a \rho g_{3}}{E} \quad \hat{M}_{i}=\frac{M_{i}}{E a^{3}} \quad \hat{T}=\alpha \Delta T \\
& \varepsilon_{11}\left(\hat{x}_{1}, \hat{x}_{2}, \hat{x}_{3}\right)=\hat{T}-\frac{v \hat{F}_{3}}{\hat{A}}-v \hat{g}_{3}\left(\hat{c}-\hat{x}_{3}\right)+ \\
& {\left[\hat{M}_{2}+\hat{F}_{1}\left(\hat{c}-\hat{x}_{3}\right)\right] \frac{v \hat{x}_{1}}{\hat{I}}+\left[-\hat{M}_{1}+\hat{F}_{2}\left(\hat{c}-\hat{x}_{3}\right)\right] \frac{v \hat{x}_{2}}{\hat{I}}} \\
& \varepsilon_{33}\left(\hat{x}_{1}, \hat{x}_{2}, \hat{x}_{3}\right)=\hat{T}+\frac{\hat{F}_{3}}{\hat{A}}+\hat{g}_{3}\left(\hat{c}-\hat{x}_{3}\right)- \\
& {\left[\hat{M}_{2}+\hat{F}_{1}\left(\hat{c}-\hat{x}_{3}\right)\right] \frac{\hat{x}_{1}}{\hat{I}}-\left[-\hat{M}_{1}+\hat{F}_{2}\left(\hat{c}-\hat{x}_{3}\right)\right] \frac{\hat{x_{2}}}{\hat{I}}} \\
& \varepsilon_{13}\left(\hat{x}_{1}, \hat{x}_{2}, \hat{x}_{3}\right)=-\frac{\hat{M}_{3} \hat{x}_{2}}{2 \hat{G} J}-\frac{\hat{F}_{2}}{2 \hat{I}}\left(v_{1}+\frac{2 v_{2} \hat{b}^{2}}{\hat{r}^{4}}\right) \hat{x}_{1} \hat{x}_{2}+ \\
& \frac{\hat{F}_{1}}{2 \hat{I}}\left[-\frac{v}{2}\left(\hat{x}_{1}^{2}-\hat{x}_{2}^{2}\right)-\frac{1}{4}\left(3 \hat{x}_{1}^{2}+\hat{x}_{2}^{2}\right)+v_{2}\left(1+\hat{b}^{2}+\frac{\hat{b}^{2}}{\hat{r}^{2}}-\frac{2 \hat{b}^{2} \hat{x}_{1}^{2}}{\hat{r}^{4}}\right)\right] \\
& \varepsilon_{23}\left(\hat{x}_{1}, \hat{x}_{2}, \hat{x}_{3}\right)=\frac{\hat{M}_{3} \hat{x}_{1}}{2 \hat{A} J}-\frac{\hat{F}_{1}}{2 \hat{A}}\left(v_{1}+\frac{2 v_{2} \hat{b}^{2}}{\hat{r}^{4}}\right) \hat{x}_{1} \hat{x}_{2}+ \\
& \frac{\hat{F}_{2}}{2 \hat{I}}\left[-\frac{v}{2}\left(\hat{x}_{2}^{2}-\hat{x}_{1}^{2}\right)-\frac{1}{4}\left(3 \hat{x}_{2}^{2}+\hat{x}_{1}^{2}\right)+v_{2}\left(1+\hat{b}^{2}+\frac{\hat{b}^{2}}{\hat{r}^{2}}-\frac{2 \hat{b}^{2} \hat{x}_{2}^{2}}{\hat{r}^{4}}\right)\right] \\
& \varepsilon_{22}\left(\hat{x}_{1}, \hat{x}_{2}, \hat{x}_{3}\right)=\varepsilon_{11}\left(\hat{x}_{1}, \hat{x}_{2}, \hat{x}_{3}\right) \quad \varepsilon_{12}\left(\hat{x}_{1}, \hat{x}_{2}, \hat{x}_{3}\right)=0
\end{aligned}
$$




$$
\begin{array}{llll}
\hat{A}=\pi\left(1-\hat{b}^{2}\right) & \hat{I}=\frac{\pi}{4}\left(1-\hat{b}^{4}\right) & v_{1}=v+\frac{1}{2} & \hat{r}^{2}=\hat{x}_{1}+\hat{x}_{2} \\
\hat{J}=\frac{\pi}{2}\left(1-\hat{b}^{4}\right) & \hat{G}=\frac{1}{2(1+v)} & v_{2}=\frac{3}{4}+\frac{v}{2} & v_{5}=v+\frac{2}{3}
\end{array}
$$

Thestress components at the surface of thecylinder can be calculated from these strains using the Duhamel-Neumann constitutive law of thermo-elasticity. If the applied surface tractions correspond in magnitude and distribution to these stresses, the solution is exact everywhere. However, if the tractions are arbitrary this solution is valid only in the Saint Venant sense. ${ }^{8}$ That is, far from the cylinder faces the solution for arbitrary end loads exponentially approaches this answer. Therefore, this solution can be considered exact to some precision for arbitrary end loading as long as it is used some distance from the cylinder faces.

\section{Measurement Techniques}

There are numerous methods to measure the properties of a deforming body. ${ }^{3}$ To further complicate matters, the selection of a measurement path is strongly intertwined with the technique. For the sake of brevity the construction of a load transducer using two types of sensors will be explored. A very general technique to infer the global state of a device is to measure the local strains by the use of electrical-resistance strain gages. Using this common electrical method as a baseline for comparison, the use of a fiber-optic differential displacement sensor will also be examined.

\section{Electrical Resistance Strain Gage}

The resistance $R$, of a conductive material attached to a strained device by a specified path is given by eq (4). ${ }^{3}$ The path is parameterized by the distance along the path, $s$. Here $A_{g}$ is the cross-sectional area of the strain gage. $\rho_{g}$ is the resistivity and $S_{g}$ is the strain sensitivity of the gage material. $\varepsilon(s)$ is the normal strain along the path of integration. This equation assumes that the strain and the gage cross-sectional area are small. That is, $|\varepsilon(s)| \leq 10^{-3}$ and $A g$ $\ll d^{2}$, where $d$ is the half-length of the gage.

$$
R(\varepsilon)=\int_{-d}^{d} \frac{\rho_{g}}{A_{g}}\left[1+S_{g} \varepsilon(s)\right] d s
$$

The actual strain measurement comes from determining the change in resistance of the gage from its initial state. This change in resistance due to a strain $R_{\Delta}(\varepsilon)$, where $R_{\Delta}(\varepsilon)=R(\varepsilon)-R(0)$, is frequently obscured due to other spurious effects such as temperature induced strain and the connecting lead resistance. ${ }^{9}$ This small resistance change is also imbedded in a large initial resistance $R(0)$, where $R_{\Delta}(\varepsilon) \ll R(0)$. To determine this small change in resistance and to account for some of the false signals, the resistance is typically found through a voltage measurement of a balanced resistance bridge circuit. 3 ,

For two strain gages that are identical except that they are placed at two different locations, the measured change in voltage can be written as in eq $(5) .{ }^{3}$ Here $S_{b}=1 / 4$ is the proportionality constant of the bridge circuit, and $\bar{\varepsilon}$ is the average strain seen by each integration path as given in eq (6).

$$
\begin{gathered}
\hat{V}_{\Delta}\left(\bar{\varepsilon}_{1} \bar{\varepsilon}_{2}\right) \approx S_{b} S_{g}\left(\bar{\varepsilon}_{1}-\bar{\varepsilon}_{2}\right) \\
\bar{\varepsilon}=\frac{1}{2 d} \int_{-d}^{d} \varepsilon(s) d s
\end{gathered}
$$

Part of the versatility of an electrical resistance technique is that the gage path can be quite general. Zigzagging paths are frequently employed to increase the sensor length in a small neighborhood on the device. Varying the cross-sectional area allows the region of greatest sensitivity to be localized to the area of maximum strain.

The sensitivity of this electrical technique for a given maximum strain, can be augmented by increasing both the bridge-excitation voltage, $V$, and the strain sensitivity, $S_{g}$. Unfortunately both of these parameters have restrictions. The bridge-excitation voltage is limited by several concerns such as heat dissipation or common mode isolation. ${ }^{9}$ The strain sensitivity is a material property and is thus limited by known materials. ${ }^{3}$

\section{Fiber-optic Differential Displacement Gage}

An alternative to the above resistive technique is a measurement based on optical wave guides. ${ }^{10} \mathrm{~A}$ fiber can be used to measure displacements or other phenomenon by various means. ${ }^{11}$ One such displacement measuring method is to configure two fibers in the form of a MachZehnder interferometer to make a 'fiber optic strain gage., The change in phase of the light between two axially strained fibers is given in eq $(7) .{ }^{12}$

$$
\varphi_{\Delta}=S_{f}\left(\vec{u}_{1}-\vec{u}_{2}\right)
$$

$\vec{u}$ is the displacement of the fiber as given in eq (8) and $d$ is the fiber half-length. The phase sensitivity of the fiberoptic measurement, $S_{f}$, is dependent on the material properties of the fiber and the wavelength of light being employed. For the present purposes this sensitivity is taken as a given parameter and is good to first order in strain. ${ }^{13}$

The sensitivity of this technique can be increased by using a short wavelength of light. At the same time this particular fiber-optic method has a geometric property the resistive technique lacks. Namely, for a fixed change in phase as the measurement path is increased the strain sensitivity will also increase.

$$
\bar{u}=\int_{-d}^{d} \varepsilon(s) d s=2 \bar{\varepsilon} d
$$


Unfortunately, this scaling of the strain sensitivity with the distance has a price. These fibers have other limitations that are not found in their electrical cousins. In particular, fibers in general have very restrictive path requirements (e.g. minimum radii of curvature limitation) to maintain light in the wave guide. ${ }^{10}$ Consequently the zigzagging paths (paths that are for practical purposes discontinuous in the first derivative of position with respect to the path length) are not allowed. In addition this interferometric sensor is no longer a state device. The sensor can only detect a relative change in phase. That is, the interferometric output beyond a single fringe is not one-to-one invertible to the strain. External means such as counters or phase trackers are required in order to integrate the change in phase. ${ }^{11,12,14,15}$

\section{Further Discussion}

Neither the electrical nor fiber-optic sensors chosen for this study are necessarily optimal. For instance restricting the electrical measurement to a half-bridge circuit will not be necessary for the measurement of the twisting moment; a full-bridge circuit could be employed. Similarly, restricting the fiber-optic technique to a Mach-Zehnder interferometer is accompanied by the problem of lead sensitivity. Other fiber-optic interferometric techniques that can relieve this lead problem might be employed. ${ }^{16}$ The primary reason for choosing the half-bridge and Mach-Zehnder techniques is that they are mathematically similar in that they both employ two differential measurement paths. This differential property will allow a natural means to subtract measured quantities. Designing for these two techniques illustrates the flavor of finding completely separable measurement paths. Focusing on these two methods here is not to suggest that others systems might not be equally useful.

\section{Measurement Paths}

Even with the restriction of the designated two types of sensors there are innumerable choices for the measurement paths. It is conceivable that the paths can traverse any part of the cylinder. Again for the sake of simplicity, the measurement paths will be assumed to be restricted to the cylinder surface. Clearly the sensors need to be located away from the cylinder faces, to allow the arbitrary end loading to approach the exact solution. Also the paths will be further restricted to the outside lateral surface (i.e., $\hat{r}=1$ ) due to its guaranteed accessibility.

The strain along the measurement path can be rewritten in terms of the components of the strain tensor and properties of the path (e.g. local unit tangent vector). ${ }^{7}$ Here the Cartesian components of the position vector $\hat{p}_{1}(s)(i=1,2,3)$ are required.

Two families of paths are used to separate the load components. In the case of fiber-optic sensors they provide for a potential increase in measurement precision. The chief attribute of these paths is that they are reasonably easy to integrate for the average strain. The helixes can be used almost exclusively except for some of the fiber-optic cases.

\section{Helical Paths}

A family of helicoids on the surface of the cylinder are parameterized in terms of the angle $\delta$ as in eq (9). The term $\hat{y}$ gives the location of the center of the path in the threedirection. The term $\phi$ indicates the angular position of the center of the measurement path in the plane of the crosssection. The symbol $\beta$ represents the angle of the helix.

$$
\begin{aligned}
& \hat{p}_{1}(\delta, \phi)=\cos (\delta+\phi) \quad \hat{p}_{3}(\delta, \hat{y}, \beta)=\hat{y}+\tan (\beta) \delta \\
& \hat{p}_{2}(\delta, \phi)=\sin (\delta+\phi)
\end{aligned}
$$

Since the helicoids are parameterized in terms of the angle $\delta$ instead of the arc length $s$, it is convenient to rewrite eq (6) in terms of $\delta$ as shown in eq (11). Here the subtended angle $\delta_{h}$ is defined in terms of the nondimensional halflength, $\hat{t}_{t}$ in eq $(10)$.

$$
\begin{gathered}
\delta_{h}=\cos (\beta) \hat{d}_{h} \\
\bar{\varepsilon}_{h}\left(\hat{d}_{h}, \phi, \hat{y}, \beta\right)=\frac{\sec (\beta)}{2 \hat{d}_{h}} \int_{-\delta_{h}}^{\delta_{h}} \varepsilon(\delta, \phi, \hat{y}, \beta) d \delta
\end{gathered}
$$

Combining eqs (9)-(11), and integrating leads to expression (12) for the average strain for the helical paths; $v_{3}$ and $v_{4}$ are defined in eq (13).

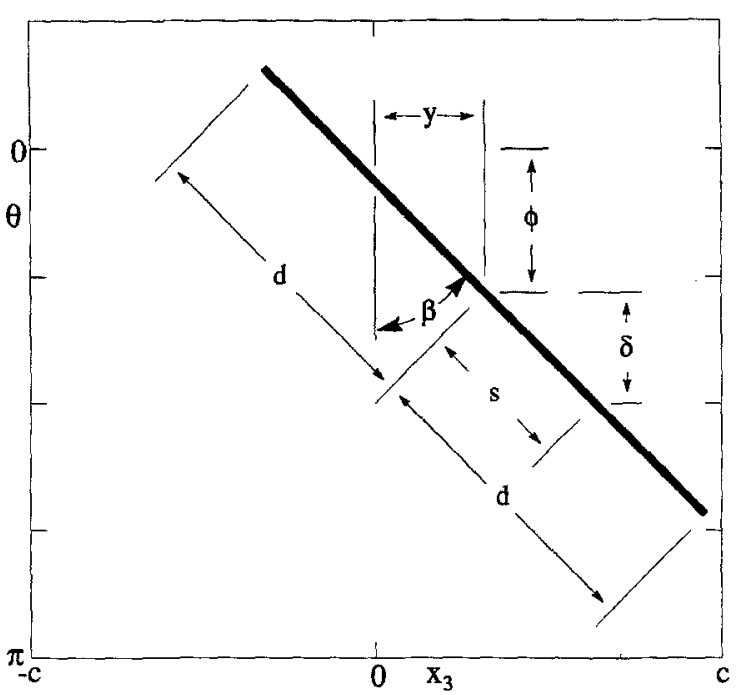

Fig. 2-Schematic of the terms used to located a measurement path on the outside lateral surface of the cylinder 


$$
\begin{gathered}
\bar{\varepsilon}_{h}\left(\hat{d}_{h, \phi}, \hat{y}, \beta\right)=\hat{T}+v_{3}\left[\frac{\hat{F}_{3}}{\hat{A}}+\hat{g}_{3}(\hat{c}-\hat{y})\right] \frac{\hat{M}_{3} \sin (\beta)}{\hat{\hat{G} J} \sec (\beta)}+ \\
\frac{v_{3}}{\hat{I}}\left\{\left[\hat{F}_{2} v_{4}-\hat{M}_{2}-\hat{F}_{1}(\hat{c}-\hat{y})\right] \frac{\sin \left(\delta_{h}\right)}{\delta_{h}}-\hat{F}_{2} \frac{\cos \left(\delta_{h}\right)}{\cot (\beta)}\right\} \cos (\phi)- \\
\frac{v_{3}}{\hat{I}}\left\{\left[\hat{F}_{1} v_{4}-\hat{M}_{1}+\hat{F}_{2}(\hat{c}-\hat{y})\right] \frac{\sin \left(\delta_{h}\right)}{\delta_{h}}-\hat{F}_{1} \frac{\cos \left(\delta_{h}\right)}{\cot (\beta)}\right\} \sin (\phi)
\end{gathered}
$$

$$
v_{3}=\frac{\tan (\beta)^{2}-v}{\tan (\beta)^{2}+1} \quad v_{4}=\tan (\beta)\left[1+\frac{\left(v_{1}+2 v_{2} \hat{b}^{2}\right)}{\left[\tan (\beta)^{2}-v\right]}\right]
$$

\section{Elliptical Paths}

The second family of measurement paths are ellipses. The ellipses are parameterized in terms analogous with the helix.

$$
\begin{aligned}
& \hat{p}_{1}(\delta, \phi)=\cos (\delta+\phi) \quad \hat{p}_{3}(\delta, \phi, \hat{y}, \beta)=\hat{y}+\tan (\beta) \sin (\delta) \\
& \hat{p}_{2}(\delta, \phi)=\sin (\delta+\phi)
\end{aligned}
$$

The elliptical path can be considered to be formed from the intersection of a plane with the outside cylindrical surface of the tube. $\beta$ is the angle between the normal of this plane and the $x_{3}$ direction. Notably here the relationship between the differential length element and differential angle is not constant as given in eq (15).

$$
d s=\sqrt{1+\tan ^{2}(\beta) \cos ^{2}(\delta) d \delta}
$$

The relationship between the half-length of the elliptical path $\hat{d}_{e}$ and the subtended angle $\delta_{e}$ is given by eq (16). The function $\mathbf{E}(\varphi, k)$ is the elliptic integral of the second kind. ${ }^{17}$

$$
\hat{d}_{e}=\sec (\beta) \mathbf{E}\left(\delta_{e}, \sin (\beta)\right)
$$

Combining eqs (14)-(16), integrating and simplifying, results in the average strain for the elliptical paths. This result is given in eq (18). The function $\mathbf{F}(\varphi, k)$ is the elliptic integral of the first kind, ${ }^{17}$ and the function as $(\varphi, \alpha)$ is defined in eq (17).

$$
\text { as }(\varphi, \alpha) \equiv \sin ^{-1}(\sin (\varphi) \sin (\alpha))
$$

$$
\begin{aligned}
& \bar{\varepsilon}_{e}\left(\hat{d}_{e}, \phi, \hat{y}, \beta\right)=\hat{T}+\frac{\hat{M}_{3}}{\hat{d}_{e} \hat{G} J}\left(\operatorname{as}\left(\delta_{e}, \beta\right)\right)+\frac{1}{\hat{d}_{e}}\left[\frac{\hat{F}_{3}}{\hat{A}}+\hat{g}_{3}(\hat{c}-\hat{y})\right] \\
& \text { - }\left[\sec (\beta) \mathbf{E}\left(\delta_{e}, \sin (\beta)\right)-\cos (\beta)(1+v) \mathbf{F}\left(\delta_{e}, \sin (\beta)\right)\right] \\
& +\frac{1}{\hat{d}_{e}}\left\{\left[\hat{M}_{2}+\hat{F}_{1}(\hat{c}-\hat{y})\right] \frac{\cos (\phi)}{\hat{I}}+\left[-\hat{M}_{1}+\hat{F}_{2}(\hat{c}-\hat{y})\right] \frac{\sin (\phi)}{\hat{I}}\right\} \\
& \text { - }\left\{\left[v_{1}+\frac{\tan ^{2}(\beta)}{2}\right] \frac{\operatorname{as}\left(\delta_{e}, \beta\right)}{\tan (\beta)}-\frac{\sin \left(\delta_{e}\right) \sqrt{1+\tan ^{2}(\beta) \cos ^{2}\left(\delta_{e}\right)}}{2}\right\} \\
& +\frac{1}{\hat{d}_{e}}\left[\hat{F}_{2} \frac{\cos (\phi)}{\hat{I}}-\hat{F}_{1} \frac{\sin (\phi)}{\hat{I}}\right] \\
& \text { - }\left\{-\frac{\tan (\beta)}{3} \sin \left(\delta_{e}\right) \cos \left(\delta_{e}\right) \sqrt{1+\tan ^{2}(\beta) \cos ^{2}\left(\delta_{e}\right)}\right. \\
& +\left[v+v_{1}+2 v_{2} \hat{b}^{2}+\frac{\left[2+\tan ^{2}(\beta)\right]}{3}\right] \csc (\beta) \mathbf{E}\left(\delta_{e}, \sin (\beta)\right) \\
& \left.-\left[\left(v_{1}+2 v_{2} \hat{b}^{2}\right) \cos ^{2}(\beta) v_{5}\right] \csc (\beta) \mathbf{F}\left(\delta_{e}, \sin (\beta)\right)\right\}
\end{aligned}
$$

\section{Signal Combinations}

These families of paths can now be combined to yield the resultant load components acting on the positive face of the cylinder. To provide a basis for comparison, the application of the electrical and optical measurement techniques will be explored independently.

\section{Electrical-resistance Strain Gages}

Motivated by eq (5), the voltage measurement in terms of the strain of the helix is given in eq (19). The half-length of all the sensors are chosen to be constant and denoted by $\hat{d}_{h}$. The center position and helical angle can be varied to provide different sensitivities to the six-load components. The primed and double primed symbols are merely dummy variable to define the function.

$\hat{V}_{\Delta}^{I}\left(\varphi^{\prime}, \phi^{\prime}, \hat{y}^{\prime}, \beta^{\prime}, \varphi^{\prime \prime}, \phi^{\prime \prime}, \hat{y}^{\prime \prime}, \beta^{\prime \prime}\right)=$

$$
S_{b} S_{g}\left[\bar{\varepsilon}_{h}\left(\hat{d}_{h}, \varphi^{\prime}+\phi^{\prime}, \hat{y}^{\prime}, \beta^{\prime}\right)-\bar{\varepsilon}_{h}\left(\hat{d}_{h, \varphi^{\prime \prime}}+\phi^{\prime \prime}, \hat{y}^{\prime \prime}, \beta^{\prime \prime}\right)\right]
$$

An example of combinations of signals using helical paths to give the resultant force and moment components on the face of cylinder are given in eqs (20)-(21). A schematic of these paths for a particular choice of parameters is shown in Fig. 3 . The parameters, $\hat{d}_{h}, \hat{y}_{1}, \phi_{1}, \phi_{2}$, and $\beta_{1}$ must be chosen such that the paths do not cross and stay near the central area of the cylinder. The requirement that the sensors do not cross is to avoid having to address this problem in a future error analysis. The end result of this procedure are the signal coefficients $C_{g i}^{d},(i=1,2, \ldots, 6)$, given in eq (22) which will determine the precision of the resultant load measurement for a given sensor arrangement. 


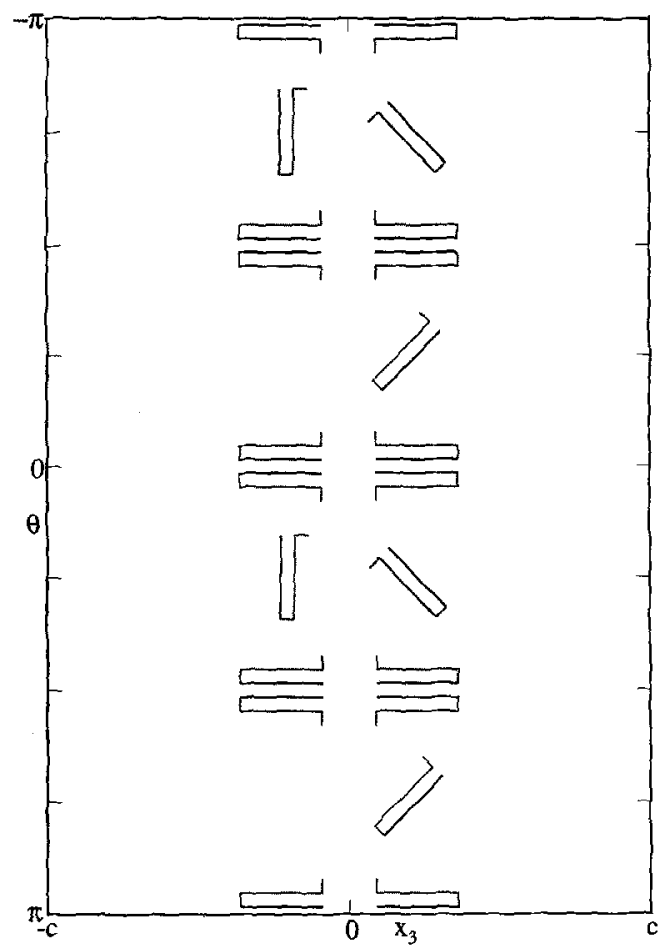

Fig. 3-Schematic of the layout of the sensor paths on the outside $(\hat{r}=1)$ cylinder surface. Referring to eqs (19)-(22) the black lines indicate signals paths with parameters $\hat{d}_{h}=(3 / 22) \hat{c}, \hat{y}_{1}=(5 / 22) \hat{c}, \phi_{1}=\pi / 16, \phi_{2}=\phi_{1}$, and $\beta_{1}=(2 / 10) \pi$. The grey lines are for additional transversely connected paths separable. In both of these cases the locations and groupings of the signals remain as specified in eqs (20) and (21). For the a sensor with cross sensitivity (i.e., a foil-type strain gage rather than a bonded wire gage $\left.{ }^{3}\right)$ the net effect on the coefficients in eq (22) is that in some cases $S_{g}$ simply needs to be modified. The zigzagging paths greatly complicate the coefficients while retaining the aforementioned favorable qualities. Unfortunately space does not permit the inclusion of this work here.

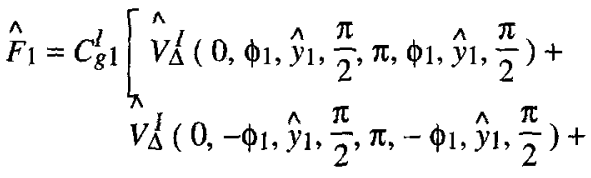

$$
\begin{aligned}
& \hat{V}_{\Delta}^{\prime}\left(\pi, \phi_{1}, \stackrel{\wedge}{-y_{1}}, \frac{\pi}{2}, 0, \phi_{1}, \hat{-}-y_{1}, \frac{\pi}{2}\right)+ \\
& \left.\hat{V}_{\Delta}^{l}\left(\pi,-\phi_{1},-y_{1}, \frac{\pi}{2}, 0,-\phi_{1}, \hat{-y_{1}}, \frac{\pi}{2}\right)\right] \\
& \hat{F}_{2}=C_{g 1}^{I}\left[\hat{V} V_{\Delta}^{I}\left(\frac{\pi}{2}, \phi_{1}, \hat{y}_{1}, \frac{\pi}{2}, \frac{3 \pi}{2}, \phi_{1}, \hat{y}_{1}, \frac{\pi}{2}\right)+\right. \\
& \hat{V}_{\Delta}\left(\frac{\pi}{2},-\phi_{1}, \hat{y}_{1}, \frac{\pi}{2}, \frac{3 \pi}{2},-\phi_{1}, \hat{y}_{1}, \frac{\pi}{2}\right)+ \\
& \hat{V_{\Delta}}\left(\frac{3 \pi}{2}, \phi_{1}, \hat{-y_{1}}, \frac{\pi}{2}, \frac{\pi}{2}, \phi_{1}, \hat{-y_{1}}, \frac{\pi}{2}\right)+ \\
& \left.\hat{V}_{\Delta}^{I}\left(\frac{3 \pi}{2},-\phi_{1},-y_{1}, \frac{\pi}{2}, \frac{\pi}{2},-\phi_{1},-y_{1}, \frac{\pi}{2}\right)\right] \\
& \hat{F}_{3}=C_{g 2}^{I}\left[\hat{V_{\Delta}^{I}}\left(0, \phi_{1}, \hat{-y_{1}}, \frac{\pi}{2}, \frac{\pi}{4}-\dot{\phi}_{2}, \phi_{2},-\hat{y}_{1}, 0\right)+\right. \\
& \hat{V_{\Delta}}\left(\pi, \phi_{1}, \hat{-} y_{1}, \frac{\pi}{2},-\frac{3 \pi}{4}-\phi_{2}, \phi_{2}, \hat{\left.-y_{1}, 0\right)}\right]+ \\
& C_{g 3}^{I}\left[\begin{array}{c}
\hat{V_{\Delta}}\left(0, \phi_{1}, \hat{y}_{1}, \frac{\pi}{2}, 0, \phi_{1},-y_{1}, \frac{\pi}{2}\right)+ \\
\hat{V_{\Delta}^{I}}\left(\pi, \phi_{1}, \hat{y}_{1}, \frac{\pi}{2}, \pi, \phi_{1}, \hat{y}_{1}, \frac{\pi}{2}\right)
\end{array}\right] \\
& \hat{M}_{1}=C_{g 4}^{I}\left[\hat{V}_{\Delta}^{I}\left(\frac{\pi}{2}, \phi_{1}, \hat{y}_{1}, \frac{\pi}{2}, \frac{3 \pi}{2}, \phi_{1}, \hat{y}_{1}, \frac{\pi}{2}\right)+\right. \\
& \left.\hat{V}_{\Delta}^{I}\left(\frac{\pi}{2},-\phi_{1}, \hat{y}_{1}, \frac{\pi}{2}, \frac{3 \pi}{2},-\phi_{1}, \hat{y}_{1}, \frac{\pi}{2}\right)\right]+ \\
& C_{g 5}^{I}\left[\hat{V_{\Delta}}\left(\frac{\pi}{2}, \phi_{1},-y_{1}, \frac{\pi}{2}, \frac{3 \pi}{2}, \phi_{1},-y_{1}, \frac{\pi}{2}\right)+\right. \\
& \left.\hat{V}_{\Delta}^{I}\left(\frac{\pi}{2},-\phi_{1},-y_{1}, \frac{\pi}{2}, \frac{3 \pi}{2},-\phi_{1},-y_{1}, \frac{\pi}{2}\right)\right] \\
& \hat{M}_{2}=C_{g 4}^{I}\left[\hat{V}_{\Delta}^{1}\left(\pi ; \phi_{1}, \hat{y}_{1}, \frac{\pi}{2}, 0, \phi_{1}, \hat{y}_{1}, \frac{\pi}{2}\right)+\right. \\
& \left.\hat{V}_{\Delta}^{I}\left(\pi,-\phi_{1}, \hat{y}_{1}, \frac{\pi}{2}, 0,-\phi_{1}, \hat{y}_{1}, \frac{\pi}{2}\right)\right]+ \\
& C_{g 5}^{I}\left[\hat{V_{\Delta}}\left(\pi, \phi_{1}, \hat{-y_{1}}, \frac{\pi}{2}, 0, \phi_{1}, \hat{-y_{1}}, \frac{\pi}{2}\right)+\right. \\
& \left.\hat{V}_{\Delta}^{I}\left(\pi,-\phi_{1},-y_{1}, \frac{\pi}{2}, 0,-\phi_{1},-y_{1}, \frac{\pi}{2}\right)\right] \\
& \hat{M}_{3}=C_{g 6}^{I}\left[\hat{V}_{\Delta}^{I}\left(\frac{\pi}{4}-\phi_{2}, \phi_{2}, \hat{y}_{1}, \beta_{1}, \frac{3 \pi}{4}-\phi_{2}, \phi_{2}, \hat{y}_{1},-\beta_{1}\right)+\right. \\
& \left.V_{\Delta}^{I}\left(-\frac{3 \pi}{4}-\phi_{2}, \phi_{2}, \hat{y}_{1}, \beta_{1},-\frac{\pi}{4}-\phi_{2}, \phi_{2}, \hat{y}_{1},-\beta_{1}\right)\right]
\end{aligned}
$$




$$
\begin{gathered}
C_{g 1}^{I}=\frac{\hat{I}}{8 S_{b} S_{g} \hat{y}_{1} \cos \left(\phi_{1}\right)} \quad C_{g^{4}}^{I}=\left(\hat{y_{1}}+\hat{c}\right) C_{g^{1}}^{I} \\
C_{g 2}^{I}=\frac{\hat{A}}{2 S_{b} S_{g}(1+v)} \quad C_{g 5}^{I}=\left(\hat{y_{1}}-\hat{c}\right) C_{g 1}^{I} \\
C_{g 3}^{I}=\frac{\hat{A}\left(\hat{y}_{1}+\hat{c}\right)}{4 S_{b} S_{g} \hat{y}_{1}} \quad C_{g 6}^{I}=\frac{\hat{G J}}{4 S_{b} S_{g} \cos (\beta) \sin (\beta)}
\end{gathered}
$$

\section{Fiber-optic Differential Displacement Gage}

Motivated by eq (7) the phase, $\varphi_{\Delta}^{\prime}$, is given in terms of the average strain of the helical paths as seen in eq (23). Here it is again assumed for the sake of brevity that all the measurement paths have the same half-length $\hat{d}_{h}$. Analogous with the half-bridge measurement of the strain gages, the change in phase is determined between two fibers.

$$
\begin{aligned}
& \varphi_{\Delta}^{I}\left(\phi^{\prime}, \hat{y}^{\prime}, \beta^{\prime}, \phi^{\prime \prime}, \hat{y}^{\prime \prime}, \beta^{\prime \prime}\right)= \\
& \quad 2 S_{f} \hat{d}_{h}\left[\bar{\varepsilon}_{h}\left(\hat{d}_{h}, \phi^{\prime}, \hat{y}^{\prime}, \beta^{\prime}\right)-\bar{\varepsilon}_{h}\left(\hat{d}_{h}, \phi^{\prime \prime}, \hat{y}^{\prime \prime}, \beta^{\prime \prime}\right)\right]
\end{aligned}
$$

An example of signals using fiber-optic sensors and helical paths is shown in Fig. 4. The signals are combined as shown in eqs (24) and (25) to yield the resultant forces and resultant moments, respectively. The solutions for the signal coefficients $C_{f i}^{l}(i=1,2, \ldots, 6)$, are given in eq (26).

As with the electrical strain gages, these signal combinations yield independent measurements of each of the load components. That is, each of the coefficients do not depend on the load components leading to zero theoretical cross-talk. Unlike the electrical case, these coefficients have a length parameter $\hat{d}_{h}$ [or through eq $(10), \delta_{h 1}$ for an inclination of $\beta_{1}$ ] in the denominator. This dependence allows the length of the sensor elements to be used to vary the magnitude of the signal coefficients and thus the sensitivity of the measurement. In other words, for a fixed geometry and a fixed minimum phase, smaller load components can be measured if there is room on the cylinder surface to increase the sensor length.

The fiber-optic signal coefficients, excluding the two bending-moment measurements and the shear measurements, can in principle be made arbitrarily small by increasing the sensor length.

The signal coefficients $C_{f 4}^{I}$ and $C_{f 5}^{\prime}$ have the proper term, $\delta_{h 1}$ in the denominator, except it is embedded in a sinusoidal function. This behavior has an easy explanation. As $\delta_{h l}$ approaches $\pi / 2$ the bending moment coefficients are at their maximum sensitivity. This corresponds to the sensor covering a total subtended angle of $\pi$. The sensors traverse the region where the normal strains are nonzero for each of the respective components. As the length increases, the path is taken to the opposite side of the cylinder and the normal strains change sign. When the total subtended angle of the path covers $2 \pi$ (as shown in Fig. 4) the measured displacements cancel leading to singular coefficients. In fact this method of determining the bending moments does not have an unrestricted scaling with sensor length. In effect the bending moment sensor paths with a half-arc-length that is an integer number times $\pi$ is invariant with respect to all the resultant loads. This sensor path, while not terribly useful for measuring loads, would be useful in a situation where the effects of cylinder loading were to be excluded in favor of sensing some other phenomenon (e.g. fiber-optic gyroscopes). ${ }^{0 .}{ }^{18}$

$$
\begin{aligned}
& \hat{F}_{1}=C_{f 1}^{I}\left[\varphi_{\Delta}^{I}\left(-\frac{\pi}{2}, \hat{y}_{1}, \beta_{1}, \frac{\pi}{2}, \hat{y}_{1}, \beta_{1}\right)+\right. \\
& \varphi_{\Delta}^{\prime}\left(\frac{\pi}{2},-\hat{y}_{2},-\beta_{1} \frac{-\pi}{2},-\hat{y}_{2},-\beta_{1}\right)+ \\
& \varphi_{\Delta}^{I}\left(-\frac{\pi}{2}, \hat{-y_{1}}, \beta_{1}, \frac{\pi}{2},-y_{1}, \beta_{1}\right)+ \\
& \left.\varphi_{\Delta}^{\prime}\left(\frac{\pi}{2}, \hat{y}_{2},-\beta_{1}, \frac{-\pi}{2}, \hat{y}_{2},-\beta_{1}\right)\right] \\
& \hat{F}_{2}=C_{f 1}^{I}\left[-\varphi_{\Delta}^{I}\left(0, \hat{y}_{1}, \beta_{1}, \pi, \hat{y}_{1}, \beta_{1}\right)+\right. \\
& \varphi_{\Delta}^{I}\left(\pi, \hat{-y_{2}},-\beta_{1}, 0,-\hat{y}_{2},-\beta_{1}\right)+ \\
& \varphi_{\Delta}^{\prime}\left(0,-y_{1}, \beta_{1}, \pi,-y_{1}, \beta_{1}\right)+ \\
& \left.\varphi_{\Delta}^{I}\left(\pi, \hat{y}_{2},-\beta_{1}, 0, \hat{y}_{2},-\beta_{1}\right)\right] \\
& \hat{F}_{3}=C_{f 2}^{?}\left[\varphi_{\Delta}^{I}\left(0, \hat{y}_{1}, \beta_{1}, \pi, \hat{y}_{3}, \beta_{2}\right)+\right. \\
& \varphi_{\Delta}^{I}\left(\frac{\pi}{2},-y_{2},-\beta_{1}, \frac{-\pi}{2},-y_{3},-\beta_{2}\right)+ \\
& \varphi_{\Delta}^{\prime}\left(\pi, \hat{y}_{1}, \beta_{1}, 0, \hat{y}_{3}, \beta_{2}\right)+ \\
& \left.\varphi_{\Delta}^{I}\left(\frac{-\pi}{2},-\hat{y}_{2},-\beta_{1}, \frac{\pi}{2},-\hat{y}_{3},-\beta_{2}\right)\right]+
\end{aligned}
$$

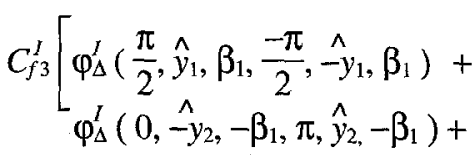

$$
\begin{aligned}
& \varphi_{\Delta}^{I}\left(\frac{-\pi}{2}, \hat{y}_{1}, \beta_{1}, \frac{\pi}{2},-y_{1}, \beta_{1}\right)+ \\
& \left.\varphi_{\Delta}^{I}\left(\pi,-y_{2},-\beta_{1}, 0, \hat{y}_{2,}-\beta_{1}\right)\right]
\end{aligned}
$$

Examining the average strain of the helical path in eq (12) for the bending moments is revealing. The only place the bending coefficients arise are in terms containing a sinusoidal change in length which is divided by a length! In effect the goal to get the bending coefficients to scale with length was doomed from the start. The average displacement of the helical paths has bending moments terms which at most scale sinusoidally with the sensor length.

An alternative approach is to sequence several portions of helical paths together such that there is a nonzero measurement as the path circles around the circumference. An example of such a sequence would be to leave the first half of the path covering a total subtended angle of $\pi$ at an inclination of $\beta_{1}$, and to make the second half of the distance at a slant of $\beta_{2}$. In effect the contribution of the negative strain could be made smaller so as to not cancel the first half of the measurement. 


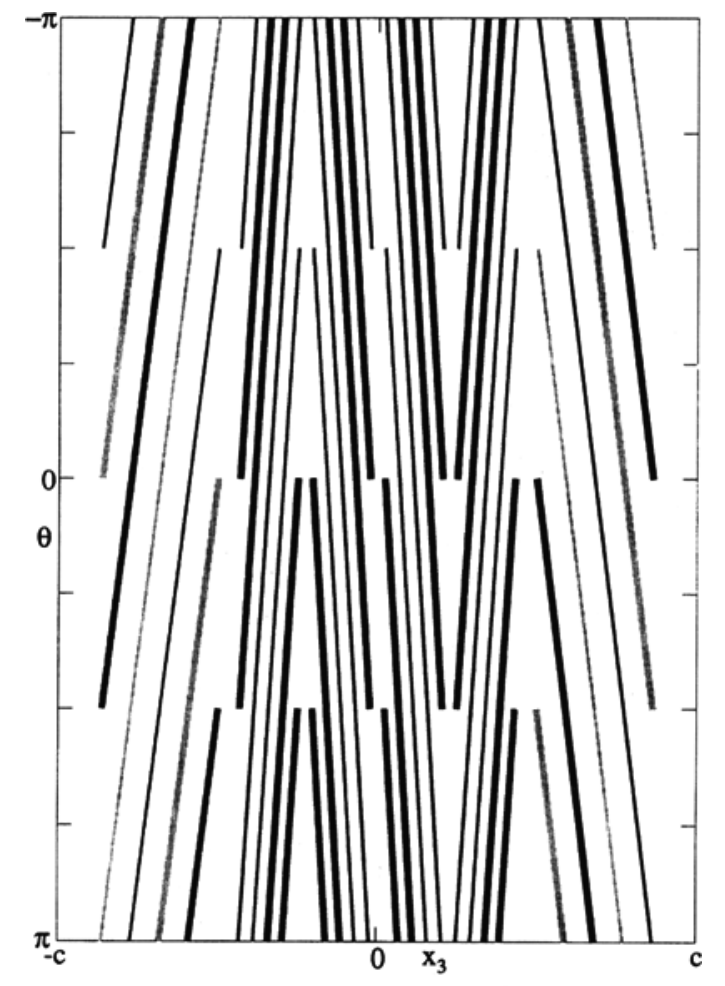

Fig. 4-Schematic of the layout of helical fiberoptic paths on the outside $(r=1)$ of the cylinder surface. Referring to eqs (23) $-(26)$ here $\delta_{h}=\pi$, $\hat{y}=(5 / 44) \hat{c}_{3}, \hat{y}_{2}=3 \hat{y}_{1}, \hat{y}_{3}=6 \hat{y}_{1}, \beta_{1}=0.014 \pi$, and $\beta_{2}=0.028 \pi$. The paths are displayed with various widths and degrees of opacity. This assists in differentiating amongst the tracks, especially at $\theta= \pm \pi$

$$
\begin{aligned}
& \hat{M}_{1}=C_{f 4}^{I}\left[\varphi_{\Delta}^{I}\left(\frac{\pi}{2}, \hat{y}_{1}, \beta_{1}, \frac{3 \pi}{2}, \hat{y}_{1}, \beta_{1}\right)+\right. \\
& \left.\varphi_{\Delta}^{I}\left(\frac{\pi}{2},-\hat{y}_{2},-\beta_{1}, \frac{3 \pi}{2},-\hat{y}_{2},-\beta_{1}\right)\right]+ \\
& C_{f 5}^{I}\left[\varphi_{\Delta}^{I}\left(\frac{\pi}{2}, \hat{-y_{1}}, \beta_{1}, \frac{3 \pi}{2},-y_{1}, \beta_{1}\right)+\right. \\
& \left.\varphi_{\Delta}^{I}\left(\frac{\pi}{2}, \hat{y}_{2},-\beta_{1}, \frac{3 \pi}{2}, \hat{y}_{2},-\beta_{1}\right)\right] \\
& \hat{M}_{2}=C_{f} I_{4}\left[\varphi_{\Delta}^{I}\left(\pi, \hat{y}_{1}, \beta_{1}, 0, \hat{y}_{1}, \beta_{1}\right)+\right. \\
& \varphi_{\Delta}^{I}\left(\pi, \stackrel{\wedge}{\left.\left.-y_{2},-\beta_{1}, 0,-\hat{y}_{2},-\beta_{1}\right)\right]+}\right. \\
& C_{f 5}^{I}\left[\varphi_{\Delta}^{I}\left(\pi, \hat{-y_{1}}, \beta_{1}, 0, \hat{-y_{1}}, \beta_{1}\right)+\right. \\
& \left.\varphi_{\Delta}^{I}\left(\pi, \hat{y}_{2},-\beta_{1}, 0, \hat{y}_{2},-\beta_{1}\right)\right] \\
& \hat{M}_{3}=C_{f 6}^{I}\left[\varphi_{\Delta}^{I}\left(0, \hat{y}_{1}, \beta_{1}, \frac{3 \pi}{2}, \hat{-y}_{2},-\beta_{1}\right)+\right. \\
& \varphi_{\Delta}^{I}\left(\pi, \hat{y}_{1}, \beta_{1}, \frac{\pi}{2},-\hat{y}_{2},-\beta_{1}\right)+ \\
& \varphi_{\Delta}^{I}\left(0,-\hat{y}_{1}, \beta_{1}, \frac{3 \pi}{2}, \hat{y}_{2},-\beta_{1}\right)+ \\
& \left.\varphi_{\Delta}^{I}\left(\pi,-\hat{y}_{1}, \beta_{1}, \frac{\pi}{2}, \hat{y}_{2},-\beta_{1}\right)\right]
\end{aligned}
$$

$$
\begin{gathered}
C_{f 1}^{I}=\left(\frac{\hat{I}}{16 \hat{d}_{h} S_{f}} \sec \left(\beta_{1}\right) \csc \left(\beta_{1}\right)\right) / \\
{\left[\cos \left(\delta_{h 1}\right)\left[v-\tan ^{2}\left(\beta_{1}\right)\right]+\frac{\sin \left(\delta_{h 1}\right)}{\delta_{h 1}}\left[\frac{1}{2}+\tan \left(\beta_{1}\right)+2 v_{2} \hat{b}^{2}\right]\right]} \\
C_{f 2}^{I}=\frac{\hat{A} \sec ^{2}\left(\beta_{1}\right) \sec ^{2}\left(\beta_{2}\right)}{8 d_{h} S_{f}(1+v)\left[\tan ^{2}\left(\beta_{1}\right)-\tan ^{2}\left(\beta_{2}\right)\right]} \\
C_{f 3}^{I}=\frac{\hat{A} \sec ^{2}\left(\beta_{1}\right)}{16 d_{h} S_{f}}\left[\frac{2 \hat{c}}{\left(\hat{y}_{2}-\hat{y}_{1}\right)\left[v-\tan ^{2}\left(\beta_{1}\right)\right]}+\right. \\
C_{f 4}^{I}=\frac{\sec ^{2}\left(\beta_{2}\right)}{16 S_{f}\left[v-\tan ^{2}\left(\beta_{1}\right)\right]\left(\hat{y}_{2}-\hat{y}_{1}\right) \sin \left(\delta_{h 1}\right) \cos \left(\beta_{1}\right)} \\
C_{f 5}^{I}=\frac{\hat{I}\left(\hat{y}_{1}+2 \hat{c}-\hat{y}_{2}\right)}{16 S_{f}\left[v-\hat{\tan }^{2}\left(\beta_{1}\right)\right]\left(\hat{y}_{2}-\hat{y}_{1}\right) \sin \left(\delta_{h 1}\right) \cos \left(\beta_{1}\right)} \\
C_{f 6}^{I}=\frac{\hat{G J}}{16 \hat{d}_{h} S_{f} \cos \left(\beta_{1}\right) \sin \left(\beta_{1}\right)}
\end{gathered}
$$

Unfortunately, this leaves the problem of connecting the two helical paths at different inclinations. Two crossing helicoids of dissimilar inclinations contained in the same cylindrical surface necessarily are not smooth at the point of intersection. Having a kink in a fiber-optic path allows light to escape the cable and disrupts the measurement. ${ }^{10}$ One could argue that in practice such a kink could be avoided and replaced with a small transition path with a suitable radius of curvature. However, this would violate the spirit of this work to exactly find separable paths. As it turns out, such an argument is not necessary.

The problem of smoothly connecting the series of paths can he solved by using a path which can intersect with the same slope. Elliptical paths have this connection capability. When half of the surface of the cylinder is traversed by an elliptical path, at the ends $\delta= \pm \delta_{e}= \pm \pi / 2$ this results in a tangent vector with a zero axial component. This tangential property allows the inclination on the path to change smoothly halfway around the cylinder from one inclination to another.

Rewriting eq (23) to incorporate the elliptical paths is shown in eq (27). $\varphi_{\Delta}^{i i}\left(\phi^{\prime}, \hat{y}^{\prime}, \beta^{\prime}, \beta^{\prime \prime}\right)$ determines the difference in phase between two fiber paths, each of which are composed of two intersecting half ellipses. The two paths are separated along the circumference of the cylinder by an angle of $\pi$. Adding a total of $m$ elements allows the path to be lengthened as shown in eq (28) $(m \geq 1)$.

$$
\begin{gathered}
\varphi_{\Delta}^{i i}\left(\phi^{\prime}, \hat{y}^{\prime}, \beta^{\prime}, \beta^{\prime \prime}\right)=2 S_{f}\left[\hat{d}_{e}\left(\beta^{\prime}\right) \bar{\varepsilon}_{e}\left(\hat{d}_{e}\left(\beta^{\prime}\right), \phi^{\prime}, \hat{y}^{\prime}, \beta^{\prime}\right)+\hat{d}_{e}\left(\beta^{\prime \prime}\right)\right. \\
\bullet \bar{\varepsilon}_{e}\left(\hat{d}_{e}\left(\beta^{\prime \prime}\right), \phi^{\prime}+\pi, \hat{y}^{\prime}+\tan \left(\beta^{\prime}\right)+\tan \left(\beta^{\prime \prime}\right) \operatorname{sgn} y^{\prime}, \beta^{\prime \prime} \operatorname{sgn} \beta^{\prime}\right) \\
-\left[d_{e}\left(\beta^{\prime}\right) \bar{\varepsilon}_{e}\left(\hat{d}_{e}\left(\beta^{\prime}\right), \phi^{\prime}+\pi, \hat{y}^{\prime}, \beta^{\prime}\right)+\hat{d}_{e}\left(\beta^{\prime \prime}\right)\right. \\
\cdot \bar{\varepsilon}_{e}\left(\hat{d}_{e}\left(\beta^{\prime \prime}\right), \phi^{\prime}+2 \pi, \hat{y}^{\prime}+\tan \left(\beta^{\prime}\right)+\right. \\
\left.\left.\tan \left(\beta^{\prime \prime}\right) \operatorname{sgn} y^{\prime}, \beta^{\prime \prime} \operatorname{sgn} \beta^{\prime}\right)\right]
\end{gathered}
$$




$$
\begin{gathered}
\varphi_{\Delta}^{I \prime}\left(m, \phi^{\prime}, \hat{y}^{\prime}, \beta^{\prime}, \beta^{\prime \prime}\right)= \\
\sum_{j=1}^{m} \varphi_{\Delta}^{i i}\left(\phi^{\prime}, \hat{y}^{\prime}+2(j-1)\left[\tan \left(\beta^{\prime}\right)+\tan \left(\beta^{\prime \prime}\right)\right] \operatorname{sgn} \hat{y}^{\prime}, \beta^{\prime}, \beta^{\prime \prime}\right)
\end{gathered}
$$

The length of each portion of the half elliptical curve is found from eq (16) and is shown in eq (29). Note that by using half-elliptical paths not only is there a smooth connection but $\delta_{e}=\pi / 2$ leads to a significant simplification to the equation for the average strain. ${ }^{18}$

$$
\begin{gathered}
\hat{d}_{e}(\beta)=\sec (\beta) \mathbf{E}\left(\frac{\pi}{2}, \sin (\beta)\right) \\
\hat{F}_{1}=C_{1}^{I I}\left[\varphi_{\Delta}^{I I}\left(m, \pi,-\hat{y}_{1},-\beta_{1}, \beta_{2}\right)+\varphi_{\Delta}^{I I}\left(m, \pi, \hat{y}_{2}, \beta_{1}, \beta_{2}\right)\right]+ \\
C_{1}^{I I}\left[\varphi_{\Delta}^{I I}\left(m, 0, \hat{y}_{1}, \beta_{1}, \beta_{2}\right)+\varphi_{\Delta}^{I I}\left(m, 0,-y_{2},-\beta_{1}, \beta_{2}\right)\right]+ \\
\hat{F}_{2}=C_{1}^{I I}\left[\varphi_{\Delta}^{I I}\left(m, \frac{\pi}{2}, \hat{y}_{1}, \beta_{1}, \beta_{2}\right)+\varphi_{\Delta}^{I I}\left(m, \frac{\pi}{2},-y_{2},-\beta_{1}, \beta_{2}\right)\right]- \\
\hat{M}_{1}^{I I}\left[\varphi_{\Delta}^{I I}\left(m,-\frac{\pi}{2},-\hat{y}_{1},-\beta_{1}, \beta_{2}\right)+\varphi_{\Delta}^{I I}\left(m,-\frac{\pi}{2}, \hat{y}_{2}, \beta_{1}, \beta_{2}\right)\right] \\
C_{4}^{I I}\left[\varphi_{\Delta}^{I I}\left(m, \frac{\pi}{2}, \hat{y}_{1}, \beta_{1}, \beta_{2}\right)+\varphi_{\Delta}^{I I}\left(m, \frac{\pi}{2},-y_{2},-\beta_{1}, \beta_{2}\right)\right]+ \\
\hat{M}_{2}=C_{4}^{I I}\left[\varphi_{\Delta}^{I I}\left(m, \frac{\pi}{2},-\varphi_{\Delta}^{I I}\left(m, \pi, \hat{y}_{1},-\beta_{1}, \beta_{2}, \beta_{2}\right)+\varphi_{\Delta}^{I I}\left(m, \frac{\pi}{2}, \hat{y}_{2}, \beta_{1}, \beta_{2}\right)\right]\right. \\
C_{5}^{I I}\left[\varphi_{\Delta}^{I I}\left(m, \pi,-,-\hat{y}_{2},-\beta_{1}, \beta_{2}\right)\right]+ \\
\end{gathered}
$$

An example of using fiber-optic sensors and a sequence of half-elliptical paths to determine the two shear load components and the two bending moment load components is shown in Fig. 5. The signals are combined as given in eq (30). The solutions for the new signals coefficients $C_{f}^{I},(i=1,4,5)$ are given in eq $(31)$.

These new coefficients now have a length parameter, $m$, in the denominator. This puts the bending moment coefficients on equal footing with the other helical path coefficients. In addition if space permitted. the sensitivity of the fiber to transverse strains could also be included. ${ }^{13}$ As with the electrical sensors these optical measurements would retain their separability and scaling with only a change to $S_{f}$.

$$
\begin{aligned}
C_{f 1}^{I I} & =\frac{\hat{I}}{8 m S_{f}\left(y_{1}-y_{2}\right)\left[g\left(\beta_{2}\right)-g\left(\beta_{1}\right)\right]} \\
C_{f 4}^{I I} & =\frac{\hat{I}\left(\hat{y}_{1}-\hat{y}_{2}+2 \hat{c}\right)}{16 m S_{f}\left(\hat{y}_{1}-\hat{y}_{2}\right)\left[g\left(\beta_{2}\right)-g\left(\beta_{1}\right)\right]} \\
C_{f}^{I I} & =\frac{\hat{I}\left(\hat{y}_{2}-\hat{y}_{1}+2 \hat{c}\right)}{16 m S_{f}\left(\hat{y_{1}}-\hat{y_{2}}\right)\left[g\left(\beta_{1}\right)-g\left(\beta_{2}\right)\right]} \\
g(\beta) & =\cos (\beta) \sin \left(\frac{\pi}{2} \cos (\beta)\right)\left[\mathrm{v}-\tan ^{2}(\beta)\right]
\end{aligned}
$$

\section{Further Discussion}

Within the confines of elastostatics of an isotropic, homogeneous, linear-elastic material the above description shows that separable component measurements exist for both electrical and optical sensors. The Mach-Zehnder optical sensors in contrast with their electrical-resistance strain-gage counterparts retain a geometric property (i.e., the sensor length) in the coefficients that determine the measurement precision. Thus a transducer designer can adjust how a fixed-phase measurement precision gets translated into a load measurement without loosing component separability or needing to increase the maximum strain.

This geometric property is double edged in the sense that if the sensor is large and there is room to extend the sensor length the measurement precision can be extended. On the other hand if the sensor needs to be small the scaling of this optical method's precision becomes worse. In this small case, the electrical gage methods might be preferred. ${ }^{3,11}$

To properly complete the design and construct this transducer an error analysis would be required. The error study would necessarily examine the effect of the fundamental assumptions as well as the precision and accuracy in which they can be implemented using mo-

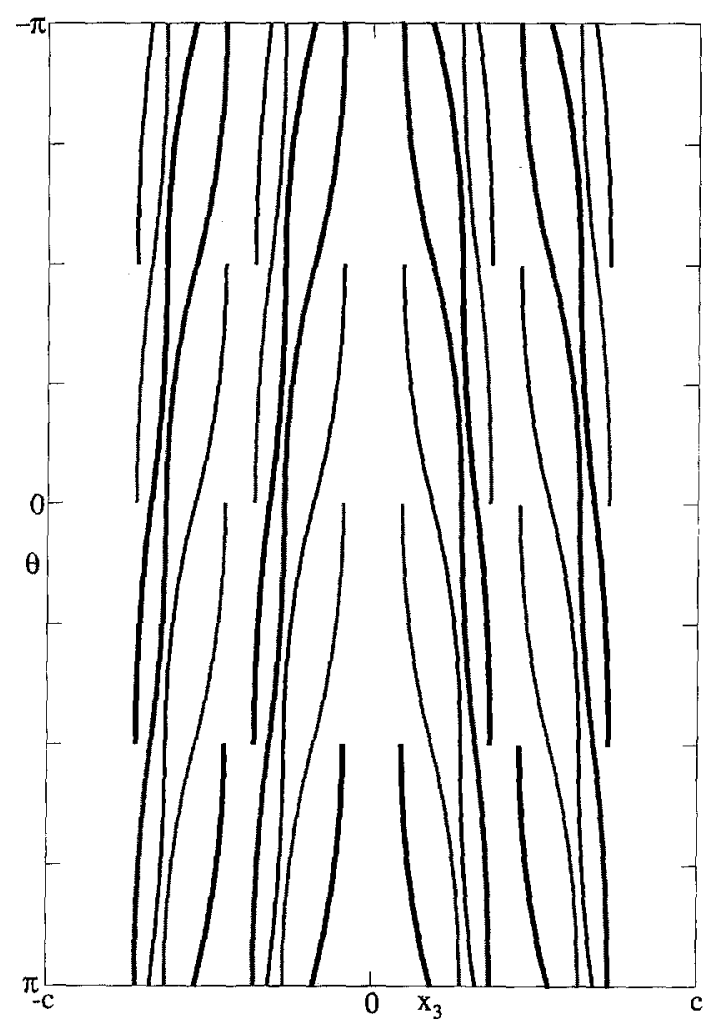

Fig. 5-Schematic of the layout of the sequence of half-elliptical fiber-optic paths on the outside surface of the cylinder. Referring to eqs (27)-(31) here $\delta_{e}=\delta_{e 1}+\delta_{e 2}=\pi / 2+\pi / 2=\pi, \hat{y}_{1}=(2 / 11) \hat{c}$, $\hat{y}_{2}=3 \hat{y}_{1}, \beta_{1}=(1 / 11) \hat{c}, \beta_{2}=(3 / 22) \hat{c}$, and $m=1$. The paths are shown with various line densities and widths to assist in differentiating amongst the tracks 
dem construction techniques. For instance the errors in position in placing long paths on the cylinder surface may accumulate for long path lengths and cause significant cross-talk. Another example would the accumulation of errors due to the wires and fibers being other than one-dimensional. ${ }^{3,13}$

The instrument must be long enough to allow the arbitrary stresses on the ends of the cylinder to decay within some precision of the exact solution. To allow for this decay the cylinder needs to be long in relation to the other dimensions (i.e., $c \gg a$, and $c \gg b$ ). Unfortunately, load transducers are typically intended to be compact and nonobtrusive to a structural system. A long-aspect ratio instrument would limit its utility. $1,2,4$

\section{Conclusions}

A six-component load transducer is described that within the confines of elastostatics has zero cross-talk amongst the resultant component measurements. This instrument can be constructed using both electrical and optical sensors of finite-measurement path length. The electrical strain gages can even have zigzagging paths and still retain the component separation. The Mach-Zehnder optical sensors allow an instrument in which the signal (i.e., total phase change) at maximum load grows with the geometry. For a fixed maximum load and signal measurement capability this geometric scaling corresponds to an increase in the precision of the load measurement.

There are some further consequences of this study. For instance, the helical paths used to measure the bending moments with fiber-optics, as discussed in 'Fiber-optic Differential Displacement Gage' above, can be made insensitive to all end loads. In effect the phase change along these paths is load invariant. Even though this sensor arrangement is not suitable for a bending-moment measurement, they would be useful for fiber-optic rotation sensors. ${ }^{18}$

A consequence of the fiber-optic geometric scaling is that the optical instruments can have lower material design strains than electrical transducers. This lower strain allows the actual instrument to be a direct load-bearing structure.

Another consequence of this geometric scaling is that the load instrument need not be constructed of structurally efficient materials. For instance, for a constant load and design strain, a lower modulus construction material would result in a bigger geometry. This larger geometry corresponds to a longer fiber-optic sensor path and thus a larger signal. Thus, constructing the sensing portion of the cylinder entirely out of glass can be considered.
Finally, making the load instrument out of a transparent material has some intriguing possibilities. Typically when imbedding fibers in a composite structure the matrix and fibers have radically different material properties. If glass fibers could he imbedded in a glass matrix, or alternatively if the index of refraction could be controlled along micron width paths through a transparent material, this would satisfy the initial material assumption for the sensing cylinder. Thus, rather than restricting the measurement paths to the cylinder surface, the entire volume of the cylinder might be utilized as a sensor.

\section{References}

1. Quinn, T.P. and Mote, C.D., "Optimal Design of an Uncoupled Six Degree of Freedom Dynamometer," EXPERIMENTAL MECHANICS, 20 (1), 40-48 (March 1990).

2. Molland, A.F., "A Five-component Strain Gauge Wind Tunnel Dynamometer," Strain, 7-13 (Jan. 1978).

3. Dally, James W. and Riley, William F., Experimental Stress Analysis, McGraw-Hill, New York, Chap. 6 (1978).

4. Holmes, J.E., "Static Multiple-Load Measurement Technique as Utilized in the Naval Surface Weapons Center's Wind Tunnels," Tech. Rep. of the Naval Surface Weapons Center 76-26, White Oak, Silver Spring, MD (April 30, 1976).

5. Timoshenko, S.P. and Goodier, J.N., Theory of Elasticity, $3 r d$ Ed., McGraw-Hill, New York (1970).

6. Butter, C.D. and Hocker, G.B., "Fiber Optics Strain Gauge," Appl. Opt., 17 (18), 2867-2869 (1978).

7. Sokolnikoff, IS., Mathematical Theory of Elasticity, Krieger Publishing, Malabar, FL 91-248 (1956).

8. Sechler, E.E., Elasticity in Engineering, Dover, New York, Section 8.2 (1952).

9. Peekel, C., "Do We Measure Strain When We Measure Strain?," Strain, 112-116 (July 1972).

10. Baker, Donald G., Monomode Fiber-Optic Design with LocalArea and Long-Haul Network Applications, Van Nostrand Reinhold, New York (1987).

11. Fiber Optic Sensors An Introduction for Engineers and Scientists, ed. E. Udd, John Wiley and Sons Inc., New York (1991).

12. Sirkis, J.S. and Taylor, C.E. "Interferometric-fiber-optic Strain Sensor," EXPERIMENTAL MECHANICS, 170-176 (June 1988).

13. Sirkis, J.S. and Haslach, H.W., "Interferometric Strain Measurement by Arbitrarily Configured, Surface-mounted, Optical Fibers," J. Lightwave Tech., 8 (10), 1497-1503 (Oct. 1990).

14. Gross, W.C., Goldstein, R., Nelson, M.D., Fearnehaugh, H.T. and Ramer, O.G., "Fiber-optic Rotation Sensor Technology," Appl. Opt., 19 (6), 852-858 (1980).

15. Kim, B.Y. and Shaw, H.J., "Phase-reading All-fiber-optic Gyroscope," Opt. Let., 9, 378 (1984).

16. Murphy, K.A., Miller, M.S., Vengsarkar, A.M. and Claus, R.O., "Elliptical-Core Two-Mode Optical-Fiber Sensor Implementation Methods," J. Lightwave Tech, 8 (11), I688-1696 (Nov. 1990).

17. Gradshteyn, I.S. and Ryzhik, I.M., Tables of Integrals, Series, and Products (Corrected and Enlarged Edition), Academic Press, New York (1980).

18. Shupe, D.M., "Fiber Resonator Gyroscope: Sensitivity and Thermal Nonreciprocity," Appl. Opt., 20 (2), 287 (Jan. 1981). 